Bull. Austral. Math. Soc.

$28 \mathrm{~A} 33,28 \mathrm{~B} 05,46 \mathrm{~A} 11,46 \mathrm{~A} 32$

VOL. 59 (1999) [449-458]

\title{
WEAK CONVERGENCE OF TENSOR PRODUCTS OF VECTOR MEASURES WITH VALUES IN NUCLEAR SPACES
}

\author{
JUN KAWABE
}

\begin{abstract}
We study weak convergence of tensor products of vector measures with values in nuclear spaces, such as the space of all rapidly decreasing, infinitely differentiable functions, the space of all test functions, and the strong duals of those spaces. It is shown that the weak convergence of a net of tensor products of vector measures follows from that of the corresponding net of real product measures.
\end{abstract}

\section{INTRODUCTION}

The notion of tensor product of vector measures was introduced by Duchon and Kluvánek [4] in 1967: Let $X$ and $Y$ be real locally convex Hausdorff spaces. Let $\mu$ be a vector measure on a measurable space $(\Omega, \mathcal{A})$ with values in $X$ and $\nu$ a vector measure on a measurable space $(\Gamma, \mathcal{B})$ with values in $Y$. Then there exists a unique vector measure $\mu \otimes \nu: \mathcal{A} \times \mathcal{B} \rightarrow X \widehat{\otimes}_{\epsilon} Y$, which is called a tensor product of $\mu$ and $\nu$, such that the relation

$$
\mu \otimes \nu(A \times B)=\mu(A) \otimes \nu(B), \quad A \in \mathcal{A}, B \in \mathcal{B}
$$

holds, where $X \hat{\otimes}_{\varepsilon} Y$ denotes the completion of the tensor product of $X$ and $Y$ under the $\varepsilon$-topology.

On the other hand, Dekiert [2] recently introduced the notion of weak convergence of vector measures with values in a Banach space, which is a natural generalisation of the weak convergence of real measures, and studied its properties (see also März and Shortt [11]).

In this paper, we study weak convergence of tensor products of vector measures with values in nuclear spaces, such as the space $\mathcal{S}$ of all rapidly decreasing, infinitely differentiable functions, the space $\mathcal{D}$ of all test functions, and the strong duals of those spaces. After preparing necessary notation and results, in Section 3 we study uniform boundedness of a set of tensor products of vector measures. In Section 4, using the results of Section 3, we show that the weak convergence of a net of tensor products of vector

\section{Received 2nd November, 1998}

This work has been supported by Grant-in-Aid for General Scientific Research No. 09640173, the Ministry of Education, Science, Sports and Culture, Japan.

Copyright Clearance Centre, Inc. Serial-fee code: 0004-9729/99 \$A2.00+0.00. 
measures with values in nuclear spaces follows from that of the corresponding net of real product measures.

All the topological spaces and topological linear spaces in this paper are Hausdorff, and the scalar fields of topological linear spaces are taken to be the field $\mathbb{R}$ of real numbers.

\section{NOTATION AND PRELIMINARIES}

Let $\langle E, F\rangle$ be a dual system over $\mathbb{R}$ with the canonical bilinear form $(x, y) \in E \times F \mapsto$ $\langle x, y\rangle$, and $\tau$ a locally convex topology on $E$ which is consistent with this duality, that is, the dual of $(E, \tau)$ is identical with $F$. The weak (respectively, strong) topology on $E$ means the $\sigma(E, F)$ (respectively $\beta(E, F)$ )-topology on $E$, and $E_{\tau}, E_{\sigma}$ and $E_{\beta}$ denote the space $E$ with the topologies $\tau, \sigma(E, F)$ and $\beta(E, F)$, respectively. If $y \in F$ and $p$ is a seminorm on $E$, we write $y \leqslant p$ whenever $|\langle x, y\rangle| \leqslant p(x)$ for all $x \in E$.

Let $(\Omega, \mathcal{A})$ be a measurable space and $\mu: \mathcal{A} \rightarrow E$ be a finitely additive set function. We say that $\mu$ is a vector measure if it is $\sigma$-additive, that is, for any disjoint sequence $\left\{A_{k}\right\}_{k=1}^{\infty}$ in $\mathcal{A}$ with $A=\bigcup_{k=1}^{\infty} A_{k}$, we have $\sum_{k=1}^{\infty} \mu\left(A_{k}\right)=\mu(A)$ for the topology $\tau$ on $E$. Denote by $\mathcal{M}(\Omega ; E)$ the set of all vector measures $\mu: \mathcal{A} \rightarrow E$. When $E=\mathbb{R}$, we write $\mathcal{M}(\Omega)$ instead of $\mathcal{M}(\Omega ; \mathbb{R})$. If $\mu$ is a vector measure, then $(y \mu)(A) \equiv\langle\mu(A), y\rangle, A \in \mathcal{A}$, is a real measure for each $y \in F$. Conversely, a theorem of Orlicz and Pettis ensures that a finitely additive set function $\mu: \mathcal{A} \rightarrow E$ is $\sigma$-additive if $y \mu$ is $\sigma$-additive for each $y \in F$.

Let $\mu: \mathcal{A} \rightarrow E$ be a finitely additive set function and $p$ be a seminorm on $E$. Then the $p$-semivariation of $\mu$ is the set function $\|\mu\|_{p}: \mathcal{A} \rightarrow[0, \infty]$ defined by

$$
\|\mu\|_{p}(A)=\sup _{y \leqslant p}|y \mu|(A), \quad A \in \mathcal{A},
$$

where $|y \mu|(\cdot)$ is the total variation of the real measure $y \mu$. See Diestel and Uhr [3] and Lewis [10] for some properties of semivariations.

Let $\mathcal{V} \subset \mathcal{M}(\Omega ; E)$. We say that $\mathcal{V}$ is uniformly bounded if $\sup _{\in \mathcal{V}}\|\mu\|_{p}(\Omega)<\infty$ for each $\tau$-continuous seminorm $p$ on $E$. Put $R(\mathcal{V}) \equiv\{\mu(A): A \in \mathcal{A}, \mu \in \mathcal{V}\} \subset E$. Then, we have a number of alternative characterisations of uniform boundedness.

Proposition 1. Let $\mathcal{V} \subset \mathcal{M}(\Omega ; E)$. Then the following conditions (a)-(d) are equivalent:

(a) $\mathcal{V}$ is uniformly bounded.

(b) For each $y \in F$, we have $\sup _{\mu \in \mathcal{V}}|y \mu|(\Omega)<\infty$.

(c) $R(\mathcal{V})$ is bounded for $\sigma(E, F)$.

(d) $R(\mathcal{V})$ is bounded for $\tau$.

Proof: $(a) \Rightarrow(b) \Rightarrow(c)$ are easy, and $(c) \Leftrightarrow(d)$ follows from [12, IV.3.3]. (a) $\Leftrightarrow$ (d) can be proved by the following inequality

$$
\sup \{p(\mu(B)): A \supset B \in \mathcal{A}\} \leqslant\|\mu\|_{p}(A) \leqslant 2 \cdot \sup \{p(\mu(B)): A \supset B \in \mathcal{A}\}
$$


Consequently, by (4)-(7), we have

$$
X_{\beta}^{*} \widehat{\otimes}_{\pi} Y_{\beta}^{*}=(X \bar{\otimes} Y)_{\beta}^{*},
$$

and the proof is complete.

We now consider tensor products of vector measures with values in dual spaces. Since $X_{\beta}^{*}$ is nuclear (see [7, Corollary 13 of I.7.B] and [14, Propositions 50.1 and 50.6]), for $\mu \in \mathcal{M}\left(\Omega ; X_{\beta}^{*}\right)$ and $\nu \in \mathcal{M}\left(\Gamma ; Y_{\beta}^{*}\right)$, there exists a tensor product $\mu \otimes \nu \in \mathcal{M}(\Omega \times$ $\Gamma ; X_{\beta}^{*} \widehat{\otimes}_{\pi} Y_{\beta}^{*}$ ) such that the relation (1) holds (see [4, Corollary 1]). For $\mathcal{V} \subset \mathcal{M}\left(\Omega ; X_{\beta}^{*}\right)$ and $\mathcal{W} \subset \mathcal{M}\left(\Gamma ; Y_{\beta}^{*}\right)$, we put $\mathcal{V} \otimes \mathcal{W} \equiv\{\mu \otimes \nu: \mu \in \mathcal{V}, \nu \in \mathcal{W}\} \subset \mathcal{M}\left(\Omega \times \Gamma ; X_{\beta}^{*} \widehat{\otimes}_{\pi} Y_{\beta}^{*}\right)$. Then we have the following

PROPOSITION 4. If $\mathcal{V} \subset \mathcal{M}\left(\Omega ; X_{\beta}^{*}\right)$ and $\mathcal{W} \subset \mathcal{M}\left(\Gamma ; Y_{\beta}^{*}\right)$ are uniformly bounded, then so is $\mathcal{V} \otimes \mathcal{W} \subset \mathcal{M}\left(\Omega \times \Gamma ; X_{\beta}^{*} \widehat{\otimes}_{\pi} Y_{\beta}^{*}\right)$.

Proof: Since $X_{\beta}^{*}$ is nuclear, the $\pi$ and $\varepsilon$ topologies coincide on $X_{\beta}^{*} \otimes Y_{\beta}^{*}$, and thus the result follows from Proposition 2.

EXAMPLE 1. (1) Let $S\left(\mathbb{R}^{m}\right)$ and $S\left(\mathbb{R}^{n}\right)$ be the spaces of all rapidly decreasing, infinitely differentiable functions on Euclidean spaces $\mathbb{R}^{m}$ and $\mathbb{R}^{n}$ respectively. These are examples of nuclear Fréchet spaces. The strong dual spaces $\mathcal{S}^{*}\left(\mathbb{R}^{m}\right)$ and $\mathcal{S}^{*}\left(\mathbb{R}^{n}\right)$ are called the spaces of all slowly increasing distributions. We have the canonical isomorphisms (see [14, Theorem 51.6 and its Corollary]):

$$
\mathcal{S}\left(\mathbb{R}^{m}\right) \widehat{\otimes}_{\pi} \mathcal{S}\left(\mathbb{R}^{n}\right)=\mathcal{S}\left(\mathbb{R}^{m+n}\right) \quad \text { and } \quad \mathcal{S}^{*}\left(\mathbb{R}^{m}\right) \widehat{\otimes}_{\pi} \mathcal{S}^{*}\left(\mathbb{R}^{n}\right)=\mathcal{S}^{*}\left(\mathbb{R}^{m+n}\right) .
$$

Consequently, for $\mu \in \mathcal{M}\left(\Omega ; \mathcal{S}\left(\mathbb{R}^{m}\right)\right)$ and $\nu \in \mathcal{M}\left(\Gamma ; \mathcal{S}\left(\mathbb{R}^{n}\right)\right)$, the tensor product $\mu \otimes \nu$ exists and takes values in $\mathcal{S}\left(\mathbb{R}^{m+n}\right)$. When $\mu \in \mathcal{M}\left(\Omega ; \mathcal{S}^{*}\left(\mathbb{R}^{m}\right)\right)$ and $\nu \in \mathcal{M}\left(\Gamma ; \mathcal{S}^{*}\left(\mathbb{R}^{n}\right)\right)$, then $\mu \otimes \nu$ also exists and takes values in $\mathcal{S}^{*}\left(\mathbb{R}^{m+n}\right)$.

(2) Let $U \subset \mathbb{R}^{m}$ and $V \subset \mathbb{R}^{n}$ be open sets. Denote by $\mathcal{D}(U), \mathcal{D}(V)$ and $\mathcal{D}(U \times V)$ the spaces of all test functions on $U, V$ and $U \times V$, respectively. These are examples of locally convex Hausdorff spaces whose type is a strict inductive limit of an increasing sequence of nuclear Fréchet spaces. The strong dual spaces $\mathcal{D}^{*}(U), \mathcal{D}^{*}(V)$, and $\mathcal{D}^{*}(U \times V)$ are called the spaces of all distributions. We have the canonical isomorphisms (see [14, Theorem 51.7] and Grothendieck [6, Chapter II, Section 3, $n^{\circ} 3$, p.84]):

$$
\mathcal{D}(U) \bar{\otimes} \mathcal{D}(V)=\mathcal{D}(U \times V) \quad \text { and } \quad \mathcal{D}^{*}(U \times V)=\mathcal{D}^{*}(U) \widehat{\otimes}_{\pi} \mathcal{D}^{*}(V)
$$

Consequently, for $\mu \in \mathcal{M}(\Omega ; \mathcal{D}(U))$ and $\nu \in \mathcal{M}(\Gamma ; \mathcal{D}(V))$, the tensor product $\mu \otimes \nu$ exists and takes values in $\mathcal{D}(U \times V)$. When $\mu \in \mathcal{M}\left(\Omega ; \mathcal{D}^{*}(U)\right)$ and $\nu \in \mathcal{M}\left(\Gamma ; \mathcal{D}^{*}(V)\right)$, then $\mu \otimes \nu$ also exists and takes values in $\mathcal{D}^{*}(U \times V)$. 


\section{WEAK CONVERGENCE OF TENSOR PRODUCT}

In this section, we first introduce the notion of weak convergence of vector measures with values in a sequentially complete lcHs. Let $X$ be a sequentially complete lcHs with a topology $\tau$. Let $S$ be a completely regular space and $\mathcal{B}(S)$ the $\sigma$-field of all Borel subsets of $S$. Denote by $C(S)$ the Banach space of all bounded, continuous, real valued functions on $S$ with the norm $\|f\| \equiv \sup _{s \in S}|f(s)|$. Let $\left\{\mu_{\alpha}\right\}$ be a net in $\mathcal{M}(S ; X)$ and $\mu \in \mathcal{M}(S ; X)$. The following definition of the weak convergence of vector measures is a natural generalisation of the weak convergence of real measures, and it is reduced to that of [15] in case that $X=\mathbb{R}$ and is reduced to that of [2] in case that $X$ is a Banach space. We say that $\left\{\mu_{\alpha}\right\}$ converges weakly to $\mu$ for $\tau$ if, for each $f \in C(S)$, we have

$$
\lim _{\alpha} \int_{S} f d \mu_{\alpha}=\int_{S} f d \mu \text { for the topology } \tau \text { on } X \text {. }
$$

See Lewis [10] for definition and basic properties of the integral above. We remark that every bounded, measurable, real valued functions on an arbitrary measurable space is integrable with respect to any vector measure with values in a sequentially complete $\mathrm{lcHs}$.

In the rest of this paper, as in Section 3, we assume that $X$ is a strict inductive limit of an increasing sequence $\left\{X_{n}\right\}$ of nuclear Fréchet spaces, $Y$ is a strict inductive limit of an increasing sequence $\left\{Y_{n}\right\}$ of Fréchet spaces, and $Z \equiv X \bar{\otimes} Y$ is a strict inductive limit of the increasing sequence $\left\{X_{n} \widehat{\otimes}_{\pi} Y_{n}\right\}$ of the completion of the tensor product of $X_{n}$ and $Y_{n}$ under the projective topology (see Section 3 for topological linear properties of these spaces).

In the following, let $S$ and $T$ be completely regular spaces which satisfy $\mathcal{B}(S \times T)=$ $\mathcal{B}(S) \times \mathcal{B}(T)$ (this condition is satisfied, for instance, both $S$ and $T$ are (1) separable metric spaces and (2) Suslin spaces; see Schwartz [13, p.105]). The following theorem shows that the weak convergence of a net of tensor products of vector measures with values in nuclear spaces follows from that of the corresponding net of real product measures. We recall that for $\mu \in \mathcal{M}(S ; X)$ and $\nu \in \mathcal{M}(T ; Y)$, the tensor product $\mu \otimes \nu$ exists and takes values in $Z \equiv X \bar{\otimes} Y$, and $Z_{\beta}^{*}$ can be identified with $X_{\beta}^{*} \widehat{\otimes}_{\pi} Y_{\beta}^{*}$ as a topological linear space.

THEOREM 5. Let $\left\{\mu_{\alpha}\right\} \subset \mathcal{M}(S ; X)$ and $\left\{\nu_{\alpha}\right\} \subset \mathcal{M}(T ; Y)$ be uniformly bounded nets. Let $\mu \in \mathcal{M}(S ; X)$ and $\nu \in \mathcal{M}(T ; Y)$. Assume that, for each $x^{*} \in X^{*}$ and $y^{*} \in Y^{*}$, the net $\left\{x^{*} \mu_{\alpha} \times y^{*} \nu_{\alpha}\right\}$ of real product measures converges weakly to the real product measure $x^{*} \mu \times y^{*} \nu$. Then $\left\{\mu_{\alpha} \otimes \nu_{\alpha}\right\} \subset \mathcal{M}(S \times T ; Z)$ converges weakly to $\mu \otimes \nu \in$ $\mathcal{M}(S \times T ; Z)$ for $\sigma\left(Z, Z^{*}\right)$. Further, if $Y$ is nuclear, it also converges weakly for the inductive limit topology on $Z$.

We first show Theorem 5 in the case that $X$ and $Y$ are Fréchet spaces and $X$ is nuclear. 
Theorem 6. Let $X$ be a nuclear Fréchet space and $Y$ a Fréchet space. Let $\left\{\mu_{\alpha}\right\} \subset \mathcal{M}(S ; X)$ and $\left\{\nu_{\alpha}\right\} \subset \mathcal{M}(T ; Y)$ be uniformly bounded nets. Let $\mu \in \mathcal{M}(S ; X)$ and $\nu \in \mathcal{M}(T ; Y)$. Assume that, for each $x^{*} \in X^{*}$ and $y^{*} \in Y^{*},\left\{x^{*} \mu_{\alpha} \times y^{*} \nu_{\alpha}\right\}$ converges weakly to $x^{*} \mu \times y^{*} \nu$. Then $\left\{\mu_{\alpha} \otimes \nu_{\alpha}\right\} \subset \mathcal{M}\left(S \times T ; X \widehat{\otimes}_{\pi} Y\right)$ converges weakly to $\mu \otimes \nu \in$ $\mathcal{M}\left(S \times T ; X \widehat{\otimes}_{\pi} Y\right)$ for $\sigma\left(X \widehat{\otimes}_{\pi} Y,\left(X \widehat{\otimes}_{\pi} Y\right)^{*}\right)$. Further, if $Y$ is nuclear, it also converges weakly for the projective topology on $X \widehat{\otimes}_{\pi} Y$.

Proof: Since $X$ is nuclear, $X \widehat{\otimes}_{\pi} Y=X \widehat{\otimes}_{\varepsilon} Y$ (see [14, Theorem 50.1]), and hence by Proposition 2 and uniform boundedness of $\left\{\mu_{\alpha}\right\}$ and $\left\{\nu_{\alpha}\right\}$, we have that $\left\{\mu_{\alpha} \otimes \nu_{\alpha}\right\} \subset$ $\mathcal{M}\left(S \times T ; X \widehat{\otimes}_{\pi} Y\right)$ is uniformly bounded. Therefore, we can easily show that the set

$$
W=\bigcup_{\alpha}\left\{\int_{S \times T} h d\left(\mu_{\alpha} \otimes \nu_{\alpha}\right)-\int_{S \times T} h d(\mu \otimes \nu):\|h\| \leqslant 1, h \in C(S \times T)\right\}
$$

is bounded in $X \widehat{\otimes}_{\pi} Y$.

Fix $\varepsilon>0, z^{*} \in\left(X \widehat{\otimes}_{\pi} Y\right)^{*}$, and $h \in C(S \times T)$ with $\|h\| \leqslant 1$. For each $\alpha$, we put

$$
z_{\alpha}=\int_{S \times T} h d\left(\mu_{\alpha} \otimes \nu_{\alpha}\right)-\int_{S \times T} h d(\mu \otimes \nu)
$$

and put

$$
V_{\varepsilon}=\left\{z^{*} \in\left(X \widehat{\otimes}_{\pi} Y\right)^{*}: \sup _{z \in W}\left|\left\langle z, z^{*}\right\rangle\right|<\varepsilon\right\} \text {. }
$$

Then, $V_{\varepsilon}$ is a neighbourhood of 0 for the strong topology on $\left(X \widehat{\otimes}_{\pi} Y\right)^{*}$. Since $\left(X \widehat{\otimes}_{\pi} Y\right)_{\beta}^{*}$ can be identified with $X_{\beta}^{*} \widehat{\otimes}_{\pi} Y_{\beta}^{*}$ by $\left[14\right.$, Proposition 50.7], there exists an element $z_{0}^{*}=$ $\sum_{k=1}^{m} x_{k}^{*} \otimes y_{k}^{*}\left(x_{k}^{*} \in X^{*}, y_{k}^{*} \in Y^{*}\right)$ such that $z^{*}-z_{0}^{*} \in V_{\varepsilon}$. Then, we have

$$
\sup _{\alpha}\left|\left\langle z_{\alpha}, z^{*}-z_{0}^{*}\right\rangle\right| \leqslant \sup _{z \in W}\left|\left\langle z, z^{*}-z_{0}^{*}\right\rangle\right|<\cdot \varepsilon,
$$

since $z_{\alpha} \in W$ for all $\alpha$. On the other hand, by assumption, for each $k=1,2, \ldots, m$, we have

$$
\left\langle z_{\alpha}, x_{k}^{*} \otimes y_{k}^{*}\right\rangle=\int_{S \times T} h d\left(x_{k}^{*} \mu_{\alpha} \times y_{k}^{*} \nu_{\alpha}\right)-\int_{S \times T} h d\left(x_{k}^{*} \mu \times y_{k}^{*} \nu\right) \rightarrow 0 .
$$

Consequently, by (8) and the above, we have

$$
\begin{aligned}
& \underset{\alpha}{\limsup }\left|\left\langle\int_{S \times T} h d\left(\mu_{\alpha} \otimes \nu_{\alpha}\right)-\int_{S \times T} h d(\mu \otimes \nu), z^{*}\right\rangle\right| \\
& \leqslant \lim \sup \left|\left\langle z_{\alpha}, z^{*}-z_{0}^{*}\right\rangle\right|+\limsup \left|\left\langle z_{\alpha}, z_{0}^{*}\right\rangle\right| \\
& \leqslant \varepsilon+\sum_{k=1}^{m} \limsup _{\alpha}\left|\left\langle z_{\alpha}, x_{k}^{*} \otimes y_{k}^{*}\right\rangle\right|=\varepsilon,
\end{aligned}
$$

and this implies that $\left\{\mu_{\alpha} \otimes \nu_{\alpha}\right\}$ converges weakly to $\mu \otimes \nu$ for the weak topology on $X \hat{\otimes}_{\pi} Y$. 
Assume that $Y$ is nuclear. Then $X \widehat{\otimes}_{\pi} Y$ is a nuclear Fréchet space, and hence it is a Montel space. Since $\left\{z_{\alpha}\right\}$ is contained in the bounded subset $W$ of $X \widehat{\otimes}_{\pi} Y$, noticing that the weak topology and the original topology coincide on bounded subsets of a Montel space (see $\left[8\right.$, p.229]), $\left\{z_{\alpha}\right\}$ converges to 0 for the projective topology on $X \widehat{\otimes}_{\pi} Y$.

Proof of Theorem 5: By Proposition 1, [14, Proposition 14.6] and uniform boundedness of $\left\{\mu_{\alpha}\right\}$ and $\left\{\nu_{\alpha}\right\}$, we can find $n_{0} \in \mathbb{N}$ such that $\mu_{\alpha}, \mu \in \mathcal{M}\left(S ; X_{n_{0}}\right)$ and $\nu_{\alpha}, \nu \in \mathcal{M}\left(T ; Y_{n_{0}}\right)$, and $\left\{\mu_{\alpha}\right\}$ and $\left\{\nu_{\alpha}\right\}$ are uniformly bounded in $\mathcal{M}\left(S ; X_{n_{0}}\right)$ and $\mathcal{M}\left(T ; Y_{n_{0}}\right)$, respectively. On the other hand, it is obvious that for each $x_{0}^{*} \in X_{n_{0}}^{*}$ and $y_{0}^{*} \in Y_{n_{0}}^{*},\left\{x_{0}^{*} \mu_{\alpha} \times y_{0}^{*} \nu_{\alpha}\right\}$ converges weakly to $x_{0}^{*} \mu \times y_{0}^{*} \nu$. Therefore, by Theorem $6,\left\{\mu_{\alpha} \otimes \nu_{\alpha}\right\}$ converges weakly to $\mu \otimes \nu$ for $\sigma\left(X_{n_{0}} \widehat{\otimes}_{\pi} Y_{n_{0}},\left(X_{n_{0}} \widehat{\otimes}_{\pi} Y_{n_{0}}\right)^{*}\right)$. Since the weak topology on $X_{n_{0}} \widehat{\otimes}_{\pi} Y_{n_{0}}$ is the topology induced on $X_{n_{0}} \widehat{\otimes}_{\pi} Y_{n_{0}}$ by the weak topology $\sigma\left(Z, Z^{*}\right)$, we can show that $\left\{\mu_{\alpha} \otimes \nu_{\alpha}\right\}$ converges weakly to $\mu \otimes \nu$ for $\sigma\left(Z, Z^{*}\right)$. The weak convergence for the inductive limit topology on $Z$ is shown in the same way as in Theorem 6 .

Next we study weak convergence of tensor products of vector measures with values in dual spaces. We recall that the strong dual of $Z \equiv X \bar{\otimes} Y$ can be identified with $X_{\beta}^{*} \widehat{\otimes}_{\pi} Y_{\beta}^{*}$ as a topological linear space.

THEOREM 7. Let $\left\{\mu_{\alpha}\right\} \subset \mathcal{M}\left(S ; X_{\beta}^{*}\right)$ and $\left\{\nu_{\alpha}\right\} \subset \mathcal{M}\left(T ; Y_{\beta}^{*}\right)$ be uniformly bounded nets. Let $\mu \in \mathcal{M}\left(S ; X_{\beta}^{*}\right)$ and $\nu \in \mathcal{M}\left(T ; Y_{\beta}^{*}\right)$. Assume that, for each $x \in X$ and $y \in Y,\left\{x \mu_{\alpha} \times y \nu_{\alpha}\right\}$ converges weakly to $x \mu \times y \nu$. Then $\left\{\mu_{\alpha} \otimes \nu_{\alpha}\right\} \subset \mathcal{M}\left(S \times T ; Z_{\beta}^{*}\right)$ converges weakly to $\mu \otimes \nu \in \mathcal{M}\left(S \times T ; Z_{\beta}^{*}\right)$ for $\sigma\left(Z^{*}, Z\right)$. Further, if $Y$ is nuclear, it also converges weakly for $\beta\left(Z^{*}, Z\right)$.

Proof: From Proposition 4 and uniform boundedness of $\left\{\mu_{\alpha}\right\}$ and $\left\{\nu_{\alpha}\right\}$ it follows that $\left\{\mu_{\alpha} \otimes \nu_{\alpha}\right\} \subset \mathcal{M}\left(S \times T ; Z_{\beta}^{*}\right)$ is uniformly bounded. Therefore, we can easily show that the set

$$
H=\bigcup_{\alpha}\left\{\int_{S \times T} h d\left(\mu_{\alpha} \otimes \nu_{\alpha}\right)-\int_{S \times T} h d(\mu \otimes \nu):\|h\| \leqslant 1, h \in C(S \times T)\right\}
$$

is weakly bounded in $Z^{*}$. Since $Z$ is barrelled, $H$ is an equicontinuous subset of $Z^{*}$.

Fix $\varepsilon>0, z \in Z$ and $h \in C(S \times T)$ with $\|h\| \leqslant 1$. Then $z$ is contained in some $X_{n_{0}} \widehat{\otimes}_{\pi} Y_{n_{0}}$. Since $H$ is equicontinuous, there exists a neighbourhood $W$ of 0 for the inductive limit topology on $Z$ such that $z \in W$ implies

$$
\left|\left\langle z, z^{*}\right\rangle\right| \leqslant \varepsilon \text { for all } z^{*} \in H,
$$

and then $W \cap\left(X_{n_{0}} \widehat{\otimes}_{\pi} Y_{n_{0}}\right)$ is a neighbourhood of 0 for the projective topology on $X_{n_{0}} \widehat{\otimes}_{\pi} Y_{n_{0}}$. Since $X_{n_{0}} \otimes Y_{n_{0}}$ is dense in $X_{n_{0}} \widehat{\otimes}_{\pi} Y_{n_{0}}$, we can find an element $z_{0}=$ $\sum_{k=1}^{m} x_{k} \otimes y_{k}\left(x_{k} \in X_{n_{0}}, y_{k} \in Y_{n_{0}}\right)$ such that $z-z_{0} \in W$.

For each $\alpha$, we put

$$
z_{\alpha}^{*}=\int_{S \times T} h d\left(\mu_{\alpha} \otimes \nu_{\alpha}\right)-\int_{S \times T} h d(\mu \otimes \nu) .
$$


Then $z_{\alpha}^{*} \in H$ for all $\alpha$. On the other hand, for each $k=1,2, \ldots, m$, we have $\left\langle x_{k} \otimes\right.$ $\left.y_{k}, z_{\alpha}^{*}\right\rangle \rightarrow 0$ by assumption. Consequently, by (9) and the above, we have

$$
\begin{aligned}
& \underset{\alpha}{\limsup }\left|\left\langle z, \int_{S \times T} h d\left(\mu_{\alpha} \otimes \nu_{\alpha}\right)-\int_{S \times T} h d(\mu \otimes \nu)\right\rangle\right| \\
& \leqslant \limsup _{\alpha}\left|\left\langle z-z_{0}, z_{\alpha}^{*}\right\rangle\right|+\underset{\alpha}{\lim \sup }\left|\left\langle z_{0}, z_{\alpha}^{*}\right\rangle\right| \\
& \leqslant \varepsilon+\sum_{k=1}^{m} \limsup _{\alpha}\left|\left\langle x_{k} \otimes y_{k}, z_{\alpha}^{*}\right\rangle\right|=\varepsilon,
\end{aligned}
$$

and this implies that $\left\{\mu_{\alpha} \otimes \nu_{\alpha}\right\}$ converges weakly to $\mu \otimes \nu$ for $\sigma\left(Z^{*}, Z\right)$.

Assume that $Y$ is nuclear. Then $Z$ is a Montel space. Since $\left\{z_{\alpha}^{*}\right\}$ is contained in the bounded subset $H$ of $Z^{*}$, noticing that the weak topology and the strong topology coincide on bounded subsets of the dual of a Montel space (see [14, Proposition 34.6]), we see that $\left\{z_{\alpha}^{*}\right\}$ converges to 0 for $\beta\left(Z^{*}, Z\right)$.

A subset $M$ of $\mathcal{M}(S)$ is said to be uniformly tight if for each $\varepsilon>0$, there exists a compact subset $K_{\varepsilon}$ of $S$ such that $|m|\left(S-K_{\varepsilon}\right)<\varepsilon$ for all $m \in M$. By the definition of uniform tightness and the Stone-Weierstrass theorem, it is readily seen that if $\left\{m_{\alpha}\right\} \subset$ $\mathcal{M}(S)$ and $\left\{n_{\alpha}\right\} \subset \mathcal{M}(T)$ are uniformly bounded and uniformly tight nets, and if $\left\{m_{\alpha}\right\}$ and $\left\{n_{\alpha}\right\}$ converge weakly to $m \in \mathcal{M}(S)$ and $n \in \mathcal{M}(T)$ respectively, then $\left\{m_{\alpha} \times n_{\alpha}\right\}$ converges weakly to $m \times n$. By this fact and Theorems 5 and 7 , we have

Corollory 8. Let $\left\{\mu_{\alpha}\right\} \subset \mathcal{M}(S ; X)$ and $\left\{\nu_{\alpha}\right\} \subset \mathcal{M}(T ; Y)$ be uniformly bounded nets. Let $\mu \in \mathcal{M}(S ; X)$ and $\nu \in \mathcal{M}(T ; Y)$. Assume the following two conditions:

(a) For each $x^{*} \in X^{*}$ and $y^{*} \in Y^{*},\left\{x^{*} \mu_{\alpha}\right\}$ and $\left\{y^{*} \nu_{\alpha}\right\}$ are uniformly tight.

(b) For each $x^{*} \in X^{*}$ and $y^{*} \in Y^{*},\left\{x^{*} \mu_{\alpha}\right\}$ and $\left\{y^{*} \nu_{\alpha}\right\}$ converge weakly to $x^{*} \mu$ and $y^{*} \nu$, respectively.

Then, $\left\{\mu_{\alpha} \otimes \nu_{\alpha}\right\}$ converges weakly to $\mu \otimes \nu$ for $\sigma\left(Z, Z^{*}\right)$. Further, if $Y$ is nuclear, it also converges weakly for the inductive limit topology on $Z$.

CoRollory 9. Let $\left\{\mu_{\alpha}\right\} \subset \mathcal{M}\left(S ; X_{\beta}^{*}\right)$ and $\left\{\nu_{\alpha}\right\} \subset \mathcal{M}\left(T ; Y_{\beta}^{*}\right)$ be uniformly bounded nets. Let $\mu \in \mathcal{M}\left(S ; X_{\beta}^{*}\right)$ and $\nu \in \mathcal{M}\left(T ; Y_{\beta}^{*}\right)$. Assume the following two conditions:

(a) For each $x \in X$ and $y \in Y,\left\{x \mu_{\alpha}\right\}$ and $\left\{y \nu_{\alpha}\right\}$ are uniformly tight.

(b) For each $x \in X$ and $y \in Y,\left\{x \mu_{\alpha}\right\}$ and $\left\{y \nu_{\alpha}\right\}$ converge weakly to $x \mu$ and $y \nu$, respectively.

Then, $\left\{\mu_{\alpha} \otimes \nu_{\alpha}\right\}$ converges weakly to $\mu \otimes \nu$ for $\sigma\left(Z^{*}, Z\right)$. Further, if $Y$ is nuclear, it also converges weakly for $\beta\left(Z^{*}, Z\right)$.

REMARK 1. By Dalecky and Fomin [1, Theorem III.2.2], every weakly convergent sequence of real Radon measures on an $R$-space is uniformly tight. Hence, if we assume 
that both $S$ and $T$ are $R$-spaces, then Corollaries 8 and 9 hold for the sequences $\left\{\mu_{n}\right\}$ and $\left\{\nu_{n}\right\}$ without the assumption (a) of uniform tightness. See III.2.2-2.4 of [1] for the definition and examples of an $R$-space. We note here that every $\sigma$-compact space and complete metric space is an $R$-space.

\section{REFERENCES}

[1] Yu.L. Dalecky and S. V. Fomin, Measures and differential equations in infnite-dimensional space (Kluwer Academic Publishers, Boston, 1991).

[2] M. Dekiert, Kompaktheit, Fortsetzbarkeit und Konvergenz von Vectormaßen, (Dissertation) (University of Essen, 1991).

[3] J. Diestel and J. J. Uhl, Jr., Vector measures, Amer. Math. Soc. Surveys 15 (American Mathematical Society, Providence, R.I., 1977).

[4] M. Duchon and I. Kluvánek, 'Inductive tensor product of vector-valued measures', Mat. Casopis Sloven. Akad. Vied. 17 (1967), 108-112.

[5] N. Dunford and J. T. Schwartz, Linear operators, Part 1: General theory (John Wiley \& Sons, New York, 1988).

[6] A. Grothendieck, Produits tensoriels topologiques et espaces nucléaires, Memoirs American Mathematical Society 16 (American Mathematical Society, Providence, R.I., 1955).

[7] J. Hoffmann-Jørgensen, The theory of analytic spaces (Matematisk Institut, Aarhus Universitet, Aarhus, 1970).

[8] H. Jarchow, Locally convex spaces (B.G. Teubner, Stuttgart, 1981).

[9] G. Köthe, Topological vector spaces I (Springer-Verlag, New York, 1983).

[10] D. R. Lewis, 'Integration with respect to vector measures', Pacific J. Math. 33 (1970), 157-165.

[11] M. März and R. M. Shortt, 'Weak convergence of vector measures', Publ. Math. Debrecen 45 (1994), 71-92.

[12] H. H. Schaefer, Topological vector spaces (Springer-Verlag, Berlin, Heidelberg, New York, 1971).

[13] L. Schwartz, Radon measures on arbitrary topological spaces and cylindrical measures, Tata Institute of Fundamental Research (Oxford University Press, Oxford, 1973).

[14] F. Treves, Topological vector spaces, distributions and kernels (Academic Press, New York, 1967).

[15] V. S. Varadarajan, 'Measures on topological spaces', Amer. Math. Soc. Transl. Ser. II 48 (1965), 161-228.

\footnotetext{
Department of Mathematics

Faculty of Engineering

Shinshu University

500 Wakasato Nagano 380-8553, Japan

e-mail: jkawabe@gipwc.shinshu-u.ac.jp
} 